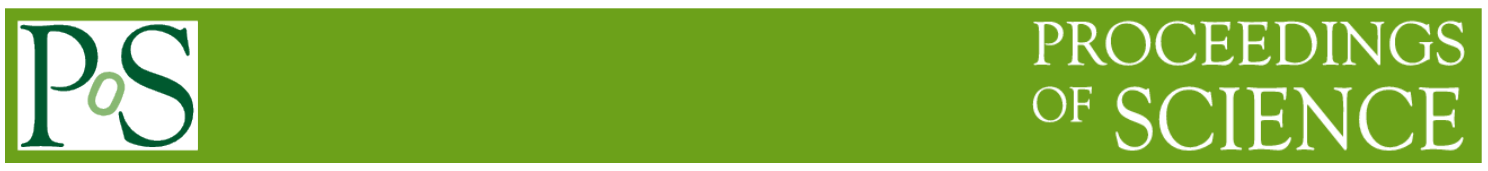

\title{
Applications of the Adelaide HEAMS muon detector.
}

\author{
Roger Clay ${ }^{1}$ \\ Department of Physics, University of Adelaide \\ North Terrace, Adelaide, Australia \\ E-mail: roger.clayeadelaide.edu.au

\section{Neville Wild} \\ Department of Physics, University of Adelaide \\ North Terrace, Adelaide, Australia \\ E-mail: neville.wildeadelaide.edu.au
}

\begin{abstract}
The Adelaide High Energy Astrophysics Muon System (HEAMS) is a sea-level spaced muon telescope consisting of four one-square-metre scintillators vertically above a second set of four. Coincidences taken with an FPGA data acquisition system (DAQ) can produce a number of directional beams and can detect coincidences from small showers, providing various useful beam energies in the range $50 \mathrm{GeV}$ to $1 \mathrm{TeV}$. Many other muon systems operating at these energies do not have the ability to record counts over such a broad range of coincidence channels.
\end{abstract}

HEAMS is located at $34^{\circ} \mathrm{S}$ and accesses an understudied declination/latitude range for these energies.

This investigation is an advanced pilot study to determine how the flexibility and power of an FPGA DAQ can enhance a muon telescope array. The detector system is described and solar and sidereal data, mainly covering mid-southern latitudes, are presented.

The 34th International Cosmic Ray Conference

30 July- 6 August, 2015

The Hague, The Netherlands

${ }^{1}$ Speaker 


\section{Introduction}

HEAMS is composed of two muon detecting systems, a simple one comprising two, one square metre, vertically spaced detectors in Adelaide and an eight detector array at the University of Adelaide field station at Buckland Park (BP) which is about $40 \mathrm{~km}$ to the north, at a latitude of $34^{\circ}$ South. Data from the detectors are available on-line, beginning in 1999. The Buckland Park system has recently been upgraded with an FPGA DAQ and is the main topic of this paper, which presents data from the first full year of the new system.

The upgraded system has multiple coincidence beams and can also identify and record coincidences produced by multiple particles in small showers, thus providing access to rate variations associated with primary particles having a range of incident energies.

\section{Hardware, beams, and pressure correction}

The HEAMS system was developed following 14 years of data taking with a one square metre coincidence system (the Adelaide detector - reported at the Rio ICRC [1]) whose data are recorded together with HEAMS (BP) data.

The Buckland Park (BP) HEAMS system is located at sea-level at a latitude of $34^{\circ}$ south. It consists of eight, one square metre, scintillators (four above four), in a light-tight, airconditioned, room with a thin roof $\left(<2 \mathrm{gcm}^{-2}\right)$. The layout is shown in figure 1 . An airconditioner maintains a stable temperature $\left( \pm 1^{\circ} \mathrm{C}\right)$, which is recorded in the data stream. The scintillators are set to trigger at the sea-level background muon rate.

Figure 1. Layout of the Buckland Park array. Four, one square metre, scintillators are directly above a similar four (900 mm vertical separation), which are spaced as shown within a light-tight room.

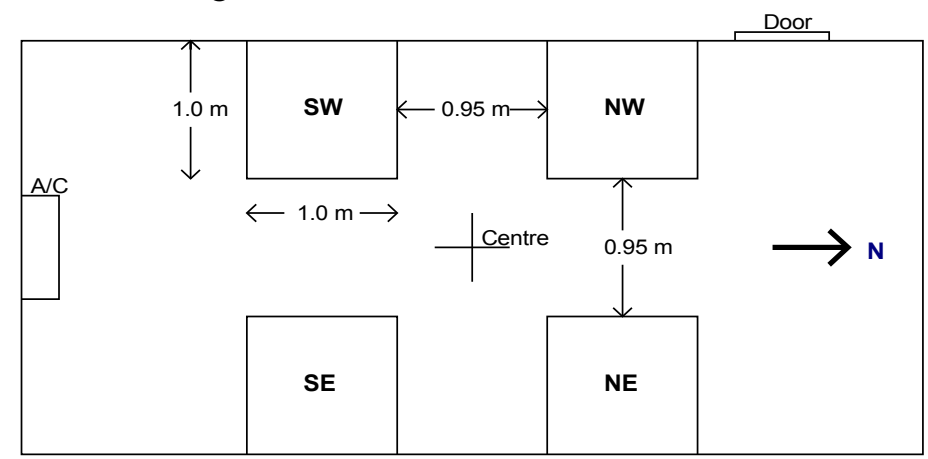

We name the individual BP detectors according to their position in the detector array e.g. UNE for Upper North East. We record single and coincidence rates at Adelaide and all the single and several different coincidence rates for BP. We do not measure pulse amplitudes. We record detector temperatures at Adelaide and BP, and barometric pressure at Adelaide, which is meteorogically close to BP.

Table 1. The (negative) pressure coefficients used in this work were (see below for explanation of the channels):

BP verticals

Adelaide Verticals

Diagonal (e.g. E-W) coinc

Vetoes

Three out of four uppers

Pressure coeff. $(\% / \mathrm{mb})$
-0.17
-0.13
-0.16
-0.4
-0.5

Note that the last two coefficients are those which would apply to small showers (which have a shorter attenuation length than muons by a factor $\sim 3$ ) whereas the first three are in the range 
expected for secondary unaccompanied muons. The coefficients are difficult to determine unambiguously and the diagonal one differs slightly $(0.16: 0.20)$ from that in [1].

All detectors use $127 \mathrm{~mm}$ Hamamatsu R877, or equivalent, photomultipliers (PMTs). Each PMT signal is pre-amplified before being connected to a discriminator which provides standard logic pulses for coincidence detection and counting. All pre-amplifiers and discriminators are home-designed and built, as is the coincidence circuitry for the Adelaide detectors. At BP we use an FPGA system for all coincidence detections and for counting. Counting at Adelaide is done using a data acquisition card installed in the HEAMS computer. The FPGA system at BP is presently configured to detect 21 different coincidences (with $25 \mathrm{~ns}$ resolving time) and to count these as well as the eight scintillator singles rates for a total of 29 (32 bit) counters. The FPGA also records and stores the total counts from the upper detectors every second in a local FIFO buffer for possible use with ground-level enhancements (GLEs) or gamma-ray burst searches. The contents of this buffer are transferred to the controlling computer every 900 seconds.

We use National Instruments (NI) hardware and software (LabVIEW) for data acquisition and control including the combining of Adelaide and BP data. The FPGA hardware at BP is an NI PCI-7811R FPGA-based DAQ, while at Adelaide we use an NI PCI-6023E data acquisition card. The FPGA is configured using LabVIEW FPGA software. We are presently using about $25 \%$ of the FPGA capability.

The scintillators are set with discrimination levels to give single muon rates of $\sim 170 \mathrm{~Hz}$ for each detector. After coincidences are taken between corresponding upper and lower scintillators, the combined BP 'vertical' rate is $\sim 225 \mathrm{~Hz}$ ( $48 \mathrm{~Hz}$ for Adelaide). Vetoes are noted (and subtracted where appropriate) when more than two scintillators trigger. The rates of coincidences of these (from small showers rather than accidentals $\sim 5 \mathrm{~Hz}$ ) and between three out of the four upper scintillators are recorded (higher energy showers $\sim 0.5 \mathrm{~Hz}$ ).

Raw, uncorrected, data are freely available on the web -

http://www.physics.adelaide.edu.au/astrophysics/muon/index.html

The purpose of the present work was to evaluate the usefulness of HEAMS for the study of cosmic rays with energies below $1 \mathrm{TeV}$ in terms of rate variations over short periods, both in solar and sidereal time.

The system should be useful because:

a. We are in a different hemisphere to most other similar systems.

b. We are at a longitude such that we see things at different times (by $10 \mathrm{~h}$ ) to the Pierre Auger Observatory scaler system [2], although at an identical latitude.

c. HEAMS responds to higher energy cosmic rays than neutron monitor systems (but note our poorer Poisson noise levels) and so adds complementary data.

\section{Short-term time variations}

The 'vertical' rates of the $\mathrm{BP}$ and Adelaide systems are approximately $225 \mathrm{~Hz}$ and $48 \mathrm{~Hz}$ rspectively. Data are recorded at $900 \mathrm{~s}$ intervals and so Poisson noise levels allow typical diurnal variations at a level of a fraction of $1 \%$ to be seen, and Forbush decreases at levels greater than $1 \%$ are clear (figures 2,3).

Figure 2 shows a web image of pressure-corrected, hourly-averaged, 'vertical' counting rates (sampled at $900 \mathrm{~s}$ intervals) for the Adelaide one square metre system and for the BP four square metre system, both expressed as percentage deviations from a nominal rate. The two systems track each other well, although the Adelaide system is in a building, located three floors 
from the roof, and should have a slightly higher energy threshold than Buckland Park. Diurnal variations at a level of $\sim 1 \%$ are clearly visible with Buckland Park Poisson noise levels being $\sim 0.2 \%$ as expected. The longer-term variations also track well, but are clearly different to neutron monitor data.

Figure 2. Web image of HEAMS data covering a recent 30 day period. Adelaide data (blue) are statistically noisier but typical variations for Adelaide and BP data clearly track each other.

Inset are 15 minute Adelaide measurements from 17:00 to $22: 45$ UTC on 22 June 2015 corresponding to the sharp peak peak which begins in the 18:30-18:45 time bin of 22/06.

(average rates are shown for 900 intervals.

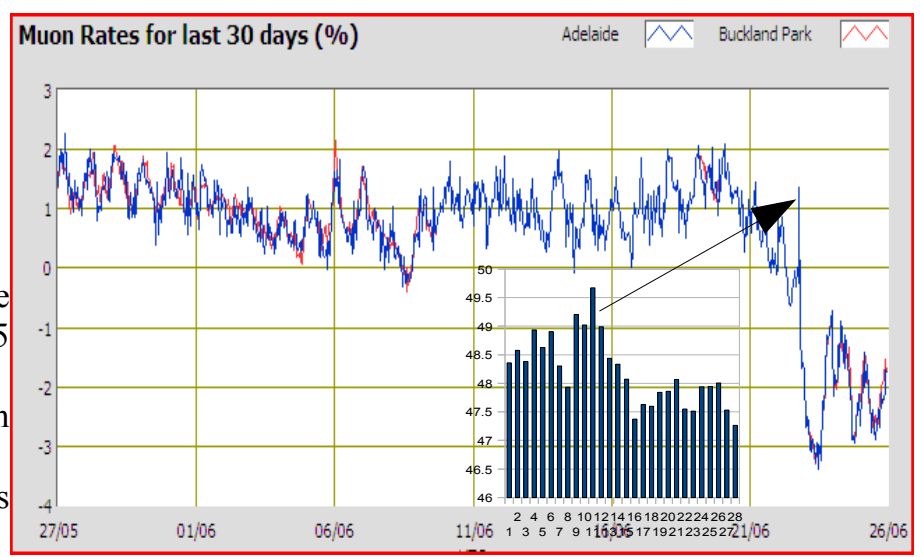

Figure 3. (a) HEAMS data (red BP, blue Adelaide - with nominal normalisation) for eight days covering the Forbush decrease of September 12 2014. The easterly (blue) and westerly (red) beams are shown in (b). Data for the same period of time from the Moscow neutron monitor are shown in (c).

[http://cr0.izmiran.rssi.ru/mosc/main.htm]

a

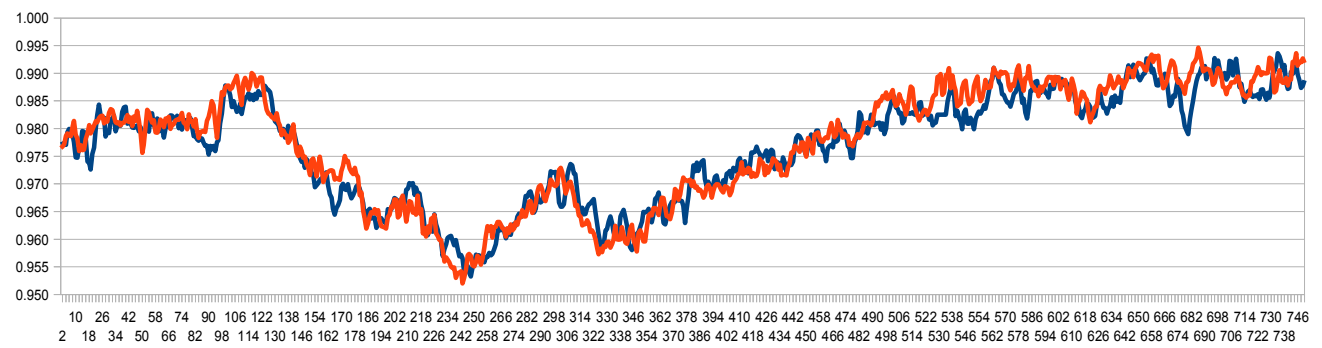

b

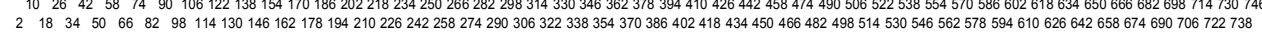

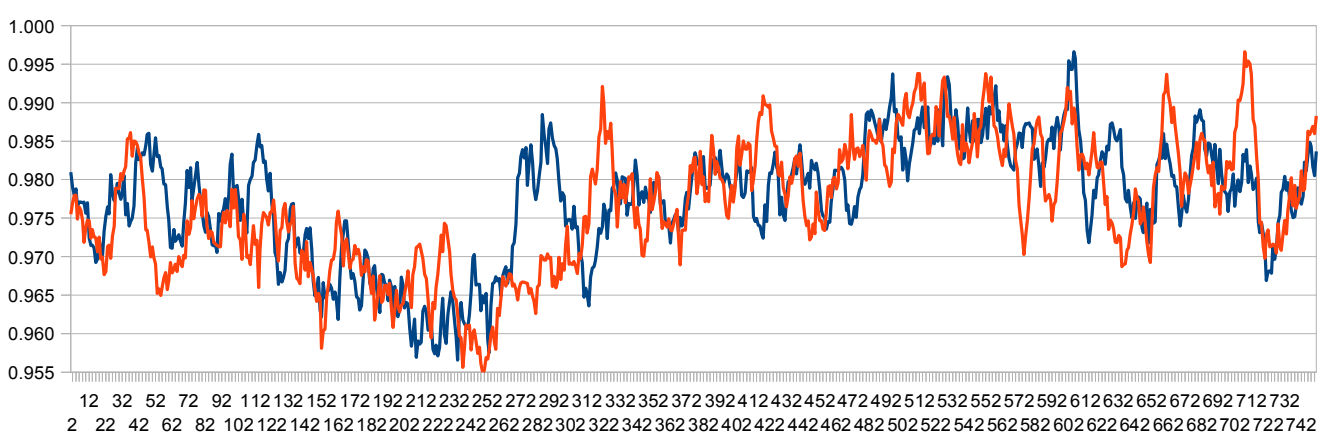

c

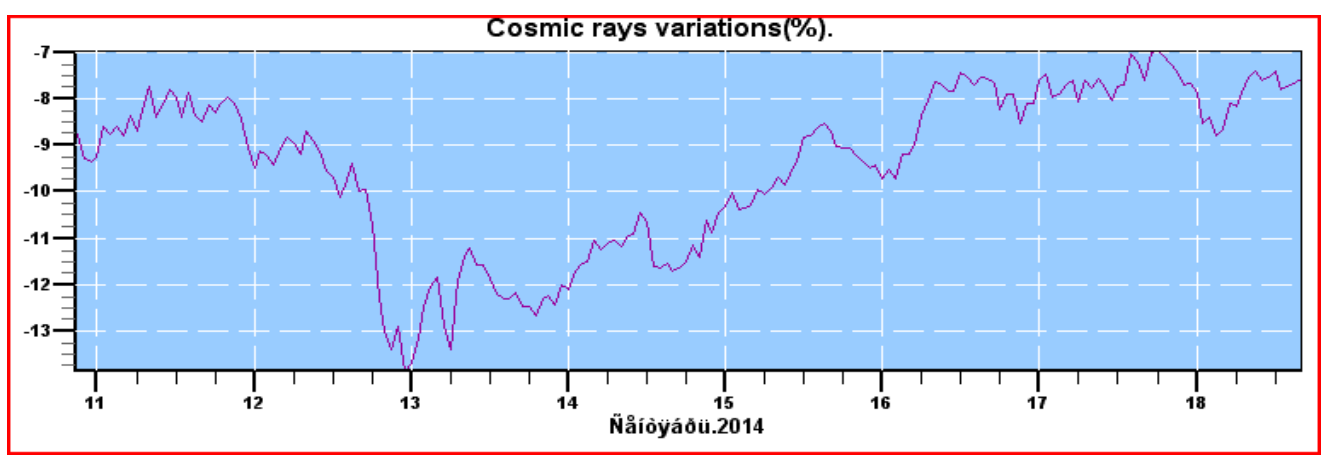




\section{Solar-time Variations}

Data folded in solar time allow one to study cosmic ray transport into the solar system as the propagation of incoming galactic cosmic rays is affected by the heliospheric magnetic field structure and the phase of the solar cycle (close to solar maximum for these data (May 2014May 2015)).

\subsection{Long-term regular solar variations :}

\subsubsection{Vertical muons}

Figure 4 shows the result of folding the BP and Adelaide vertical counts over a full solar year.

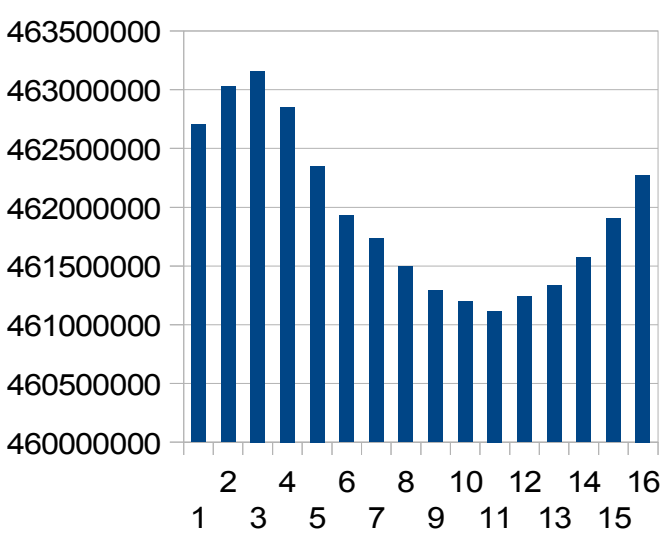

a.

Figure 4. The variation of (a) the BP 'vertical' muon rate and (b) the Adelaide rate over one solar day (totalled over a full year). Each interval is of $1.5 \mathrm{~h}$ duration in UTC (zero is 09:30 local time, with the peak at $\sim 13: 30$ local).

The solar day variations are not completely stable. Figure 5 shows the variation of the mean count rate over a solar day for a number of time periods, compared to a period of time early in May 2014 (\% difference is shown). The period 1-14 July 2014 shows little structural variation but (compared to the early May period) the other times show a difference which is approximately $12 \mathrm{~h}$ out of phase with the main component, of the nature of a second harmonic at the $0.1 \%$ level.

Figure 5. Deviations (\%) from the mean solar time variation in the period 7-14 May 2014 for a number of time periods in mid-2014.

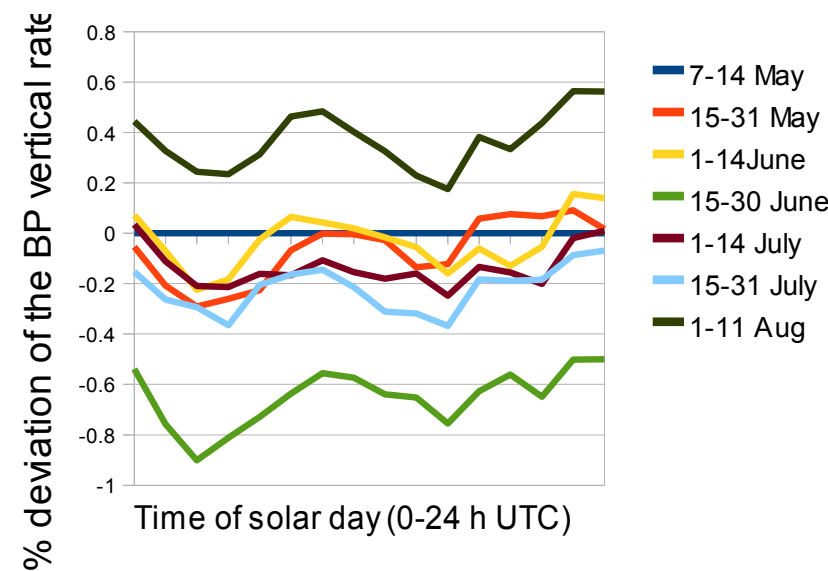




\subsubsection{Eastern beam in solar time shifted to correspond to the vertical beam.}

Increasing the zenith angle of observations increases the atmospheric depth traversed and raises the energy range under observation. HEAMS can accomplish this by taking coincidences between upper scintillators and selected lower ones. An easterly beam (mean zenith angle about $60^{\circ}$ ) results from coincidences between the two upper east scintillators and the two lower west ones (in pairs). The rate is now reduced (to $\sim 5 \mathrm{~Hz}$ ) because fewer upper scintillators are in use, the atmospheric depth is greater, and the observable solid angle is reduced. However, such coincidences are also possible for small showers and these extra coincidences are found by recording times when a further particle is in coincidence $(\sim 1 \mathrm{~Hz})$. This rate is subtracted from the east beam rate for each $900 \mathrm{~s}$ counting interval, noting that the pressure coefficient of the 'vetoes' differs from that of the muon beam.

Figure 6. The variation of the BP easterly muon rate over one solar day (totalled over a full year). Each interval is of $1.5 \mathrm{~h}$ duration in UTC. These data times have been shifted so the pointing direction corresponds to a vertical beam in timing, to correspond to the solar sky direction as in figure 4 .

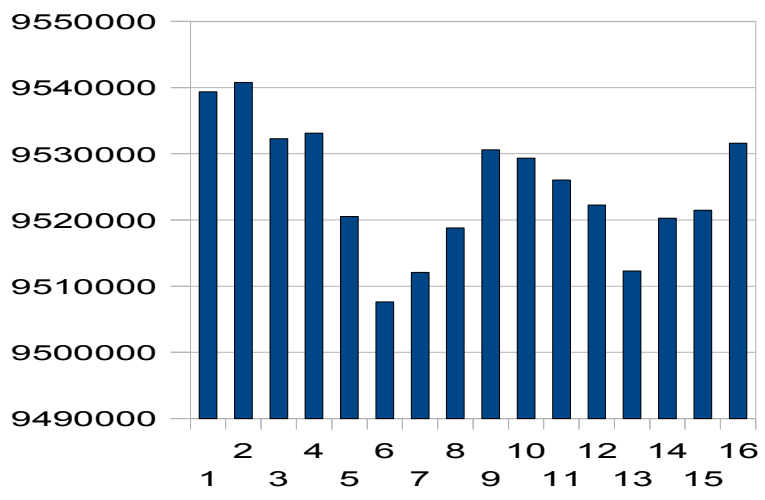

\subsubsection{North Eastern beam (cross diagonal - higher energy since higher zenith angle) full year}

A further beam is possible, that is using 'cross diagonals'. In this case, the upper north east scintillator in coincidence with the lower south west. The counting rate reduces even more $(\sim 1 \mathrm{~Hz})$, with an increase in the relative number of vetoes $(0.4 \mathrm{~Hz})$.

Figure 7. The variation of the BP northeasterly muon rate over one solar day (totalled over a full year). Each interval is of $1.5 \mathrm{~h}$ duration in UTC. These data times have been shifted so the pointing direction corresponds to a beam along the northerly meridian as in figure 4 .

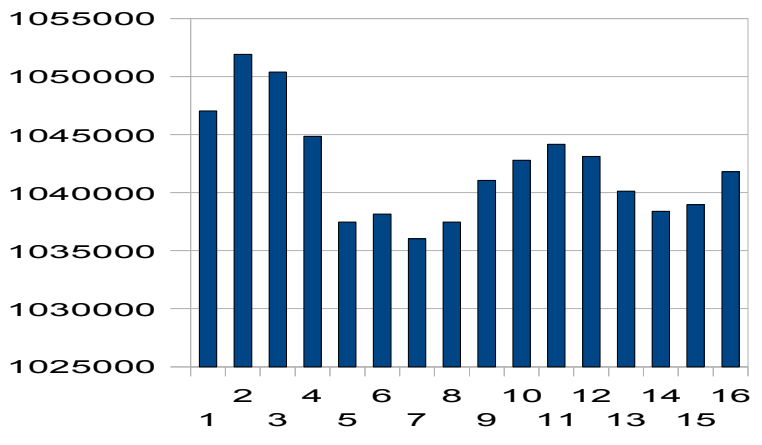

\subsubsection{Small showers (vetoes)}

Vetoes correspond to small showers and can be summed to provide a rate of showers which trigger any of the coincidence pairs plus any other scintillator (avoiding double counting). That is, the requirement is for two particles to be in a coincidence which do not make a possible single muon track.

Figure 8 . The variation of the BP summed vetoes rate rate over one solar day (totalled over a full year). Each interval is of $1.5 \mathrm{~h}$ duration in UTC.

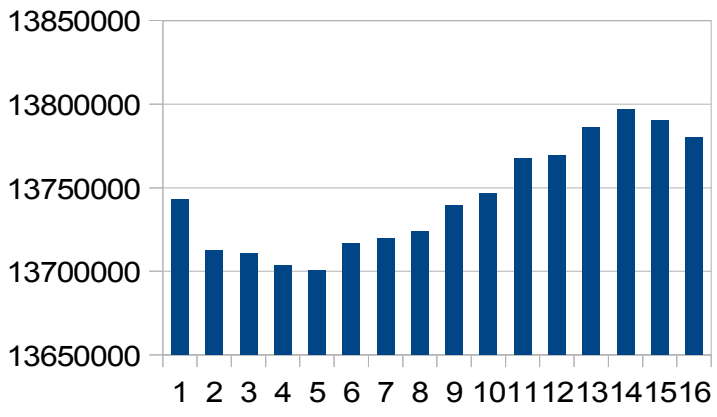


As with the solar rate for vertical coincidences, an examination was made for any deviations from this average curve. The strongest deviations (at a level of a maximum of $0.2 \%$ ) in the monthly rates of January through May 2015 were in intervals 3-8 (4.5 h to $12 \mathrm{~h} \mathrm{UTC}$ ), again centred at about $12 \mathrm{~h}$ from the main peak and suggesting the presence of a second harmonic to the solar day variation.

At higher energies $(<10 \mathrm{TeV})$ the Tibet II air shower array [3] found a solar time variation with the same phase as HEAMS but at a level almost a factor of 10 lower (fitted sinusoid with an amplitude of $0.03 \%$ ).

\subsubsection{More energetic showers (3 out of 4 upper scintillators)}

Showers with a higher primary energy can be selected by demanding that three or more detectors trigger in a single level. That is, demanding that at least three particles have been detected. HEAMS at Buckland Park has such a trigger, based on the upper group of four scintillators. The characteristic rate is now only about $0.5 \mathrm{~Hz}$. Figure 9 shows that the daily solar variation is broadly similar to that for the showers recorded by the vetoes, although with much poorer statistics

Figure 9. The variation of the rate of coincidences between the upper scintillators of the BP HEAMS system (at least three out of four required) over one solar day (totalled over a full year). Each interval is of $1.5 \mathrm{~h}$ duration in UTC.

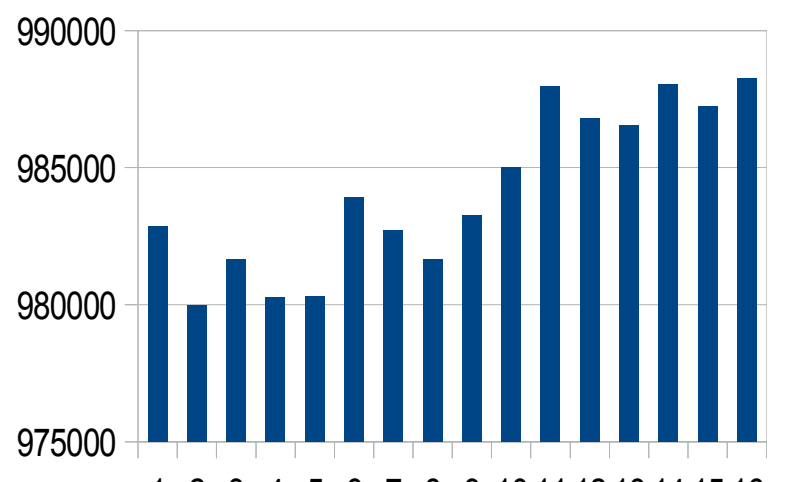

12345678910111213141516

\subsubsection{Effect of IMF Sector Boundaries}

It is possible that the polarity of the interplanetary magnetic field (IMF) sector within which cosmic ray variations are measured may influence the resulting observations. Figure 10 shows the difference between observations of the BP vertical rate within a positive IMF sector compared to a negative sector summed for the period May to December 2014. The sectors are determined from: http://www.leif.org/research/sblist.txt

Figure 10.

The difference between $\mathrm{BP}$ vertical rates (May to December 2014) for periods of $+v e$ and -ve IMF polarity, averaged over a solar day.

A sinusoidal fit to this difference has an amplitude of $0.07 \%$ and peaks at $205^{\circ}$. There is a possible corresponding variation for the the variation for the veto rate, at rather higher energies, of $0.05+/-0.05 \%$ at $280^{\circ}$.

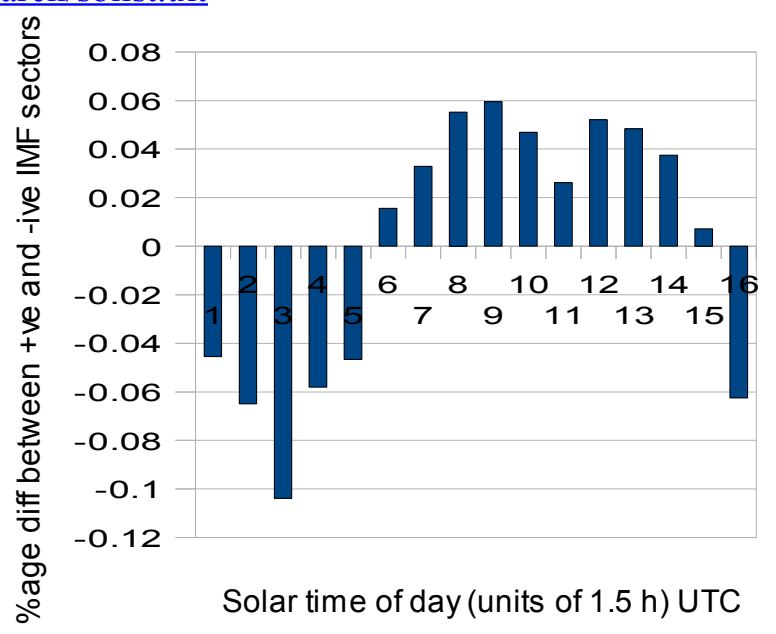

\section{Variations in sidereal time (anti-sidereal analysis):}

HEAMS data can be analysed to search for a sidereal time component in the data. This is difficult since the sidereal variation is at a lower level than that of the solar component. The 
technique of anti-sidereal analysis, first suggested by Farley and Storey [4], which requires a full year of data taking, was used to extract this component.

IceCube [5] has presented sidereal data for a similar declination to that studied here (but at energies a decade higher $-10 \mathrm{TeV}$ ). At a mean declination of $-36^{\circ}$, IceCube finds a sidereal amplitude of $9.3+/-0.5 * 10^{-4}$ and a phase of $51.2+/-3.3^{\circ}$ which is compatible with the highest energy HEAMS channel. At slightly lower energies "multi-TeV", the Tibet III air shower array [6] also finds a similar phase and comparable amplitude.

$\begin{array}{lcccc} & \text { Amp * } 10^{4} & \text { Phase }(\mathrm{deg}) & \begin{array}{c}\text { sigma stat. } \\ (\mathrm{amp} * 10000)\end{array} & \begin{array}{c}\text { Energy }(\mathrm{GeV}) \\ {[\text { Rigidity }(\mathrm{GV})]}\end{array} \\ \text { Vertical } & 5.1 & 315 & 0.1 & 60 \\ \text { High Energy } & 4.3 & 330 & 0.7 & 300 \\ \text { Very High E } & 12.2 & 53 & 2.5 & 800 \\ \text { North } & 8.0 & 11 & 2.0 & 105 \\ \text { East Beam } & 17.4 & 339 & 3.4 & 105\end{array}$

Table 2. The sidereal amplitude and phase of various beams of the Buckland Park HEAMS array corescponding to different trigger requirements and corresponding typical energies (analysed using antisidereal analysis).

\section{Conclusions}

The Adelaide University High Energy Astrophysics Muon System (HEAMS) can easily measure the daily solar time distribution of low energy cosmic rays at about $100 \mathrm{GeV}$ (single scintillators), rather higher than energies recorded by neutron monitors. This includes solar events and the flow of extra-solar particles inwards along Heliospheric magnetic fields.

It has coincidence channels which are not possible in most other muon systems operating at these energies, and shows that there is a change in the flow of cosmic rays above $100 \mathrm{GeV}$ in solar time, with an increased bi-directional flow.

Sidereal variations (in stellar coordinates) are at an appreciably lower level and are challenging for HEAMS. They are interesting but probably will not show time variations.

\section{Future work}

A result of this pilot study is that we now intend to improve our recording of small showers by adding further coincidence channels such as two- and three-fold coincidences for detectors in each of the HEAMS planes.

Work will be done to further check the pressure and temperature corrections applied to the data over longer time periods, and past solar maximum.

\section{References}

1. Berkova, M,. et al. Atmospheric Variations as observed by Adelaide and Buckland Park muon telescopes. $33^{\text {rd }}$ ICRC Rio (2013)

2. Dasso, S. and Asorey, H. for the Pierre Auger Collaboration, The scaler mode in the Pierre Auger Observatory to study the heliospheric modulation of cosmic rays. Advances in Space Research 49 (2012) 1563-1569

3. Munakata, K. et al. Solar modulation of galactic cosmic-ray anisotropy observed by the Tibet II air shower array at $\sim 10 \mathrm{TeV}$. ICRC 2001 (2001)

4. Farley, F.J.M. and Storey, J.R. The siderial variation of extensive air showers. Proc. Phys. Soc. A67 (1954) 996-1004

5. Abassi, R. et al. Observation of anisotropy in the galactic cosmic ray arrival directions at $400 \mathrm{TeV}$ with IceCube The Astrophysical Journal 746 (2012) 33 [arXiv:1109.1017]

6. Amenomori, M., et al. On temporal variations of the multi-TeV cosmic ray anisotropy using the Tibet III Air Shower Array. The Astrophysical Journal 711 (2010) 119-124

[arXiv:1001.2646] 